\title{
Preparation of Polyvinyl Alcohol/Xylan Blending Films with 1,2,3,4-Butane Tetracarboxylic Acid as a New Plasticizer
}

\author{
Cun-dian Gao, ${ }^{1}$ Jun-li Ren, ${ }^{1}$ Shuai-yang Wang, ${ }^{1}$ Run-cang Sun, ${ }^{1,2}$ and Li-hong Zhao ${ }^{1}$ \\ ${ }^{1}$ State Key Laboratory of Pulp and Paper Engineering, South China University of Technology, Guangzhou, Guangdong 510641, China \\ ${ }^{2}$ Institute of Biomass Chemistry and Technology, College of Materials Science and Technology, Beijing Forestry University, \\ Beijing 100083, China
}

Correspondence should be addressed to Jun-li Ren; renjunli@scut.edu.cn and Li-hong Zhao; zhaolh@scut.edu.cn

Received 24 January 2014; Accepted 17 February 2014; Published 27 March 2014

Academic Editor: Ming-Guo Ma

Copyright (C) 2014 Cun-dian Gao et al. This is an open access article distributed under the Creative Commons Attribution License, which permits unrestricted use, distribution, and reproduction in any medium, provided the original work is properly cited.

\begin{abstract}
Miscible, biodegradable polyvinyl alcohol (PVA)/xylan blending films were firstly prepared in the range of the PVA/xylan weight ratio from $1: 2$ to $3: 1$ by casting method using 1,2,3,4-butane tetracarboxylic acid (BTCA) as a new plasticizer. The properties of blending films as functions of PVA/xylan weight ratio and BTCA amount were discussed. XRD and FT-IR were applied to characterize the blending films. Experimental results indicated that tensile strength (TS) and elongation at break (EAB) of blending films decreased along with the decrease of the PVA/xylan weight ratio. Both of TS and EAB firstly increased and then decreased as the amount of BTCA was increased. More importantly, blending films were biodegraded almost by $41 \%$ with an addition of $10 \%$ BTCA in blending films within 30 days in soil. For all hydroxyl functionalized polymers (xylan and PVA), their molecular interactions and miscibility with BTCA endowed blending films with the biocompatibility and biodegradability. Therefore, these blending films are environmentally friendly materials which could be applied as biodegradable plastics for food packaging and agricultural applications.
\end{abstract}

\section{Introduction}

In recent years, to meet the requirements for environmental protection, multifunctional utilization of natural resources, and increased pressure from related strict laws, efforts have been made to develop biodegradable composite plastic products by incorporating biocompatible polymers as an alternative to conventional synthetic plastic materials derived from petroleum $[1,2]$. Moreover, there is an increasingly important trend to explore natural renewable raw materials for the food industries and nonfood industries owing to their sustainable and biodegradable features.

Xylan is derived from hemicellulose which is one of the three primary components of the plant cell wall [3] and many researchers paid extensive attention to the exploration of xylan because of its abundance and biodegradability. Hemicelluloses (mainly xylans and mannans) were used as sustainable packaging materials and more hydrophobic films with the low water vapor and oxygen permeability could be obtained $[4,5]$. Xylan films modified by longchain anhydride were prepared [6]. Modified xylan had better film-forming performance and the tensile strength had increased and moisture-sensitive properties had been lessened due to long carbon chains modification. Xylan has poor film-forming ability and pure xylan films were brittle due to the hydroxyl groups present in each of their repeating units [7]. To solve this problem, different types of plasticizers such as oligosaccharides, polyols, some organic acids, lipids, and ether were added in order to improve mechanical performance of biopolymers films [8-11]. It was found that mechanical properties of xylan-based films were strengthened by blending with nanosized particles [5]. More hydrophobic butylated xylan films were prepared by Peresin et al. [11]. The water sensitivity and wetting behaviour of the xylan derivatives were altered during etherification. Mikkonen et al. [12] studied arabinoxylan films plasticized with glycerol and sorbitol; they found that an increase in the total plasticizer content resulted in more permeable films. 
Polyvinyl alcohol (PVA) is excellently fit for blending with natural polymers as it is highly polar and controllable in water solutions [13]. Additionally, it is a biocompatible synthetic crystalline polymer with high tensile strength and flexibility [14]. Several investigations have been carried out on blending PVA with other biopolymers, such as PVA/chitosan [15], PVA/starch [16, 17], and PVA/gelatin [18]. Modification and biocompatibility of PVA/chitosan films were investigated by Zhuang et al. [15]. It was observed that the addition of PVA could improve the film's flexibility and wettability. All prepared PVA/chitosan films were biocompatible. Moreover, the tensile strength (TS) of the PVA/starch films increased about $60 \%$ without additives, while EAB decreased about $420.5 \%$ [17]. And the films were degraded by about $45-65 \%$ after 165 days in soil. Thus, the blending of PVA and natural polymers could improve the properties of natural polymers films for widening the application of films. However, few researchers have made attempts to study biomaterial films by blending PVA with xylan.

1,2,3,4-Butane tetracarboxylic acid (BTCA) is one of polycarboxylic acids (PCAs). BTCA as a crosslinking agent was used to produce a cotton fabric, and the treated fabric showed a reasonable hydrophobicity [19]. Water-resistant hemicelluloses (HC) films were prepared with about 10-11\% of BTCA [20]. The water vapor permeability of HC films with a modification of BTCA reduced about twice the untreated films. The effects of BTCA on hydrophobic cellulose fabric by scanning electron microscopy were investigated and the better durable fabric was obtained [21]. So the addition of BTCA could have important effects on the properties of films and fabrics.

In view of the issue mentioned above, the objective of our study was to prepare miscible and biodegradable PVA/xylan blending films by casting method with the addition of BTCA as a plasticizer for improving the properties of blending films. The influences of the PVA/xylan weight ratio and BTCA amounts on the blending films properties were discussed. The mechanical properties, water vapor permeability, the degree of solubility, thermal stability, and degradability were evaluated for PVA/xylan blending films.

\section{Experimental}

2.1. Materials. Beech wood xylan $\left(M_{w} \sim 130,000 \mathrm{~g} / \mathrm{mol}\right)$ and PVA $\left(M_{w}\right.$ of $\left.146,000 \sim 186,000 \mathrm{~g} / \mathrm{mol}\right)$ were purchased from Sigma Aldrich (Germany) and used without further purification. BTCA was supplied by Aladdin Reagent Company Limited (Shanghai, China). Anhydrous calcium chloride $\left(\mathrm{CaCl}_{2}\right)$ and sodium chloride $(\mathrm{NaCl})$ were of analyticalreagent grade and obtained from Guangzhou Chemical Reagent Factory (Guangzhou, China). Deionized water was used in the preparation of the blending films.

2.2. Blending Films Preparation. The PVA/xylan blending films were prepared by solution casting method. A predetermined amount of PVA was added to ultrapure water. The mixture was stirred for $0.5 \mathrm{~h}$ at room temperature and then heated at $95^{\circ} \mathrm{C}$ for $1.5 \mathrm{~h}$. After PVA was dissolved completely,
TABLE 1: Detailed composition and abbreviations of PVA/xylan blending films.

\begin{tabular}{lcc}
\hline Samples & PVA : xylan & BTCA $(\%)$ \\
\hline P3X1 & $3: 1$ & 20 \\
P2X1 & $2: 1$ & 20 \\
P1X1 & $1: 1$ & 20 \\
P1X2 & $1: 2$ & 20 \\
PX0 & $3: 1$ & 0 \\
PX5 & $3: 1$ & 5 \\
PX10 & $3: 1$ & 10 \\
PX15 & $3: 1$ & 15 \\
PX25 & $3: 1$ & 25 \\
PX30 & $3: 1$ & 30 \\
\hline
\end{tabular}

xylan was added. After 30 minutes, BTCA as a new plasticizer was added in the mixture solution. The resulting solution reacted at $75^{\circ} \mathrm{C}$ for $4 \mathrm{~h}$ and then poured onto a $110 \times 110 \times$ $7 \mathrm{~mm}$ teflon mould. Water was dried slowly in a ventilated oven at $40^{\circ} \mathrm{C}$ overnight. All films were obtained with a uniform thickness and were stored at $23^{\circ} \mathrm{C}$ with $50 \% \mathrm{RH}$ at least $48 \mathrm{~h}$ prior to all measurements. The blending films with different PVA/xylan weight ratio and BTCA amounts were shown in Table 1.

\subsection{Method of Characterization}

2.3.1. Tensile Strength (TS) and Elongation at Break (EAB). TS and EAB of films were evaluated using a tensile testing machine (Instron Universal Test Machine Model 5565, USA) fitted with a $100 \mathrm{~N}$ load cell. Five samples $(110 \mathrm{~mm}$ length, $150 \mathrm{~mm}$ width) of each film were cut by a paper cutter (FQQZD15, Sichuan, China). The crosshead speeds of $30 \mathrm{~mm}$ and $20 \mathrm{~mm} / \mathrm{min}$ were used for testing the mechanical properties. The maximum load and the final extension at break were used for calculation of TS and EAB, respectively.

2.3.2. Water Vapor Permeability (WVP). The WVP assessment was determined according to a gravimetric method and performed as described by Kayserilioğlu et al. [22] with minor modification. Film specimens were clamped on the top of polyethylene bottles containing $3 \mathrm{~g}$ anhydrous $\mathrm{CaCl}_{2}$ to maintain $0 \% \mathrm{RH}$ inside the bottle, followed by sealing the film over the edge of bottle with vaseline. And then the bottles were located in a dryer at $23^{\circ} \mathrm{C}$ and $75 \% \mathrm{RH}$. The $\mathrm{RH}$ was maintained by placing $500 \mathrm{~mL}$ of saturated $\mathrm{NaCl}$ solution in the bottom of the dryer. The bottle was weighed every $24 \mathrm{~h}$, and WVP value was calculated by

$$
\mathrm{WVP}=\frac{\Delta w \times L}{(\Delta t \times A)}
$$

where WVP was water vapor permeability $\left(\mathrm{g} \cdot \mu \mathrm{m} \cdot \mathrm{cm}^{-2} \cdot \mathrm{h}^{-1}\right)$. $\Delta w / \Delta t$ was the gain rate of bottle $(\mathrm{g} / \mathrm{h}) . A$ was exposed area of the film $\left(\mathrm{cm}^{-2}\right) . L$ was the mean thickness of the film $(\mu \mathrm{m})$.

2.3.3. The Water Absorption (WA), Solubility (S), and Water Content (WC). A certain quality $\left(m_{0}\right)$ of oven-dried 
PVA/xylan blending films was cut into $2 \times 2 \mathrm{~cm}^{2}$ and dipped in $20 \mathrm{~mL}$ ultrapure water for $24 \mathrm{~h}$ at room temperature. Then we wiped off the excess moisture in the surface of the swollen samples and weighed the weight $\left(m_{1}\right)$. Afterward, the soaked films were dried again at $105^{\circ} \mathrm{C}$ to the constant weight $\left(m_{2}\right)$. The water absorption (WA) and solubility $(S)$ are calculated with

$$
\begin{gathered}
\mathrm{WA}=\frac{\left(m_{1}-m_{2}\right)}{m_{0}}, \\
S(\%)=\frac{\left(m_{0}-m_{2}\right)}{m_{2}} \times 100 .
\end{gathered}
$$

Water content was determined at $23^{\circ} \mathrm{C}$ and $50 \% \mathrm{RH}$ for two days when the weight of the films was regarded as $m$. Then the samples were dried at $105^{\circ} \mathrm{C}$ to reach constant weight $\left(m_{3}\right)$. Water content $(\mathrm{WC})$ was calculated as follows:

$$
\mathrm{WC}(\%)=\frac{\left(m-m_{3}\right)}{m_{3}} \times 100 \% .
$$

2.3.4. Thermogravimetric Analysis (TGA). A simultaneous thermal analyzer (TGA Q500, TA Instruments, New Castle, USA) was used for the thermogravimetric analysis of blending films. Film samples of approximately $9 \sim 11 \mathrm{mg}$ were cut into pieces and heated at a rate of $10^{\circ} \mathrm{C} / \mathrm{min}$ from room temperature to $600^{\circ} \mathrm{C}$ under a nitrogen atmosphere (a flow rate about $20 \mathrm{~mL} / \mathrm{min}$ ). All films were conditioned at $23^{\circ} \mathrm{C} / 50 \% \mathrm{RH}$ for one week before TGA.

2.3.5. FTIR Spectra. Film samples were cut into pieces $(10 \mathrm{~mm} \times 10 \mathrm{~mm})$ and dried in the infrared drying oven. FTIR spectra were measured on a Fourier transform spectrophotometer (Nicolet 750, Florida, USA). The absorbance spectrum $\left(4000-400 \mathrm{~cm}^{-1}\right)$ was acquired at $4 \mathrm{~cm}^{-1}$ resolution and recorded for a total of 32 scans, using Attenuated Total Reflectance technique (see Figure 5).

2.3.6. X-Ray Diffraction. X-ray patterns of PVA, xylan, and $\mathrm{PVA} / \mathrm{xylan}$ blending film were analyzed using an X-ray diffractometer (Bruker, model D8 advance) with $\mathrm{Cu} \mathrm{K} \alpha$ radiation at a voltage of $40 \mathrm{kV}$ and $40 \mathrm{~mA}$. The measurements were made with scattering angles of $3-40^{\circ}$ and a scanning speed of $2^{\circ} / \mathrm{min}$. The samples were dried and stored in a desiccator before testing.

2.3.7. The Degradability of Blending Films in Soil. The degradability of blending films was determined by soil burial degradation. $3 \times 3 \mathrm{~cm}$ squares were cut from the cast film and buried in the soil at the depth of $10 \mathrm{~cm}$ in a gardening pot. The pot was exposed to atmospheric conditions for 30 days. The time required for blending films to disintegrate was recorded. To determine the weight loss in specimens, films were first dry-cleaned with a brush and the weight was recorded. A specimen of each sample was quickly cleaned and then the sample was dried in an oven at $70^{\circ} \mathrm{C}$ to constant weight and

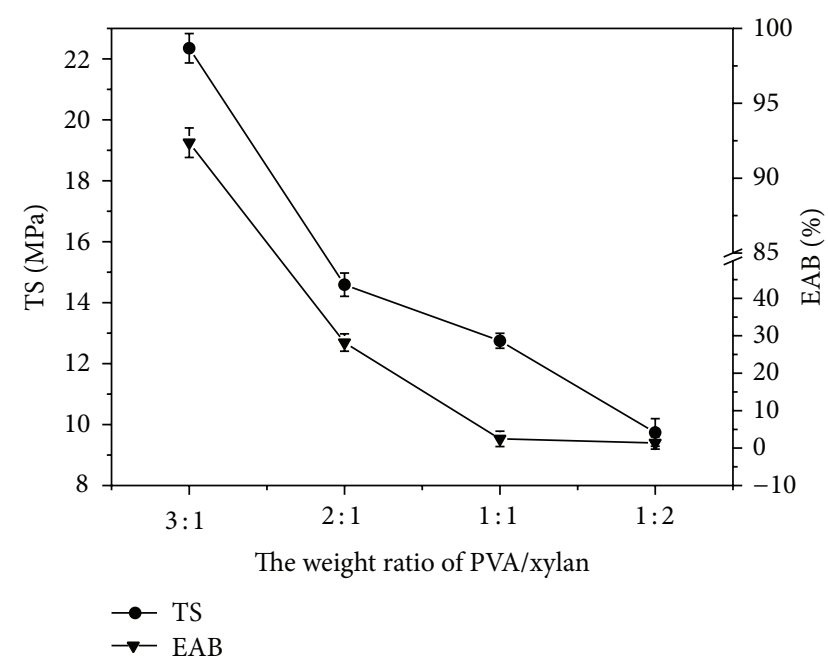

FIGURE 1: The effect of the weight ratio of PVA/xylan on TS and EAB of blending films with 20\% BTCA.

allowed to equilibrate in desiccators prior to weighing. The degradation degree (the weight loss) was determined by

$$
\text { Weight loss }(\%)=\frac{m_{\text {ini }}-m_{\text {dry }}}{m_{\text {ini }}} \times 100 \%,
$$

where $m_{\text {ini }}$ is the initial weight of the film sample before burial and $m_{\mathrm{dry}}$ is the dry weight of the sample after burial for known days.

\section{Results and Discussion}

3.1. Mechanical Properties. The thickness of blending films which has a significant influence on mechanical properties of the PVA/xylan blending films was measured by Lorentzen $\&$ Wettre thickness gauge (Sweden) for precise specimens $(55-60 \mu \mathrm{m})$. The effect of the weight ratio of PVA/xylan on TS and EAB of the blending films with the addition of $20 \%$ BTCA is shown in Figure 1. The results indicated that TS and $\mathrm{EAB}$ of the blending composite films decreased obviously along with the decrease of the weight ratio of PVA and xylan from $3: 1$ to $1: 2$. The reason explained was that PVA has high tensile strength with hydrogen bonds and xylan is brittle and amorphous with its acetyl and feruloyl groups, as well as $\alpha$-D-glucopyranosyl uronic acid or $4-\mathrm{O}$-methyl ether $[4,10,23]$. The values of TS and EAB reached to $22.4 \mathrm{MPa}$ and $92.4 \%$, respectively, as the weight ratio of PVA and xylan was $3: 1$.

Figure 2 shows the effect of BTCA content on TS and EAB of blending films at the PVA/xylan weight ratio of $3: 1$. When BTCA content was $0 \%$, the lowest value of $\mathrm{EAB}$ was obtained while relatively high TS value was present. With increasing BTCA content to 5\%, a decrease in TS and an increase in EBA were observed. With a further increment of BTCA amount from $5 \%$ to $30 \%$, both TS values and $\mathrm{EAB}$ increased first and then decreased in the range of $16.4-22.4 \mathrm{MPa}$ and $45.5 \%-$ $92.4 \%$, respectively. It could be explained that polymerpolymer interactions strengthen intermolecular forces at the 


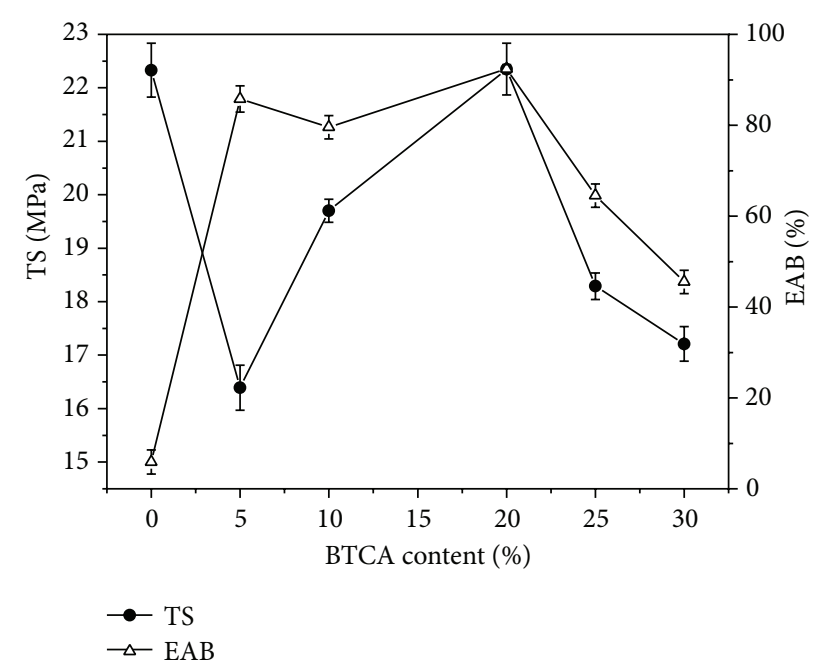

FIgUre 2: The effect of the BTCA content on TS and EAB of the blending films.

beginning of adding BTCA. Subsequently, the formation of polymer-plasticizer interactions among the macromolecules restrained intermolecular mobility and revealed the decrease of TS and EAB [24]. When BTCA amount reached 20\%, TS and $\mathrm{EAB}$ obtained their maximum values simultaneously which were higher than the PVA/polysaccharides (angico gum and/or cashew gum) blended films investigated by Silva et al. [25]. Moreover, the addition of BTCA provided the hydroxyl-rich chemicals with carboxyl functional groups. Yoon et al. [26] verified that additives of the films with hydroxyl and carboxyl groups were stronger and more flexible than those with only hydroxyl groups. Thus BTCA had the important impact on mechanical properties of PVA/xylan blending films.

3.2. WVP Measurement. WVP is an important parameter of film for potential applications such as food packaging. Figure 3 illustrates the effect of the weight ratio of PVA/xylan and the BTCA content on WVP. Clearly, WVP was decreased slightly and then increased sharply with the weight ratio of PVA/xylan changing from $3: 1$ to $1: 2$ at the $20 \%$ BTCA content. The lowest value was achieved to $0.017 \mathrm{~g} \cdot \mu \mathrm{m} \cdot \mathrm{cm}^{-2} \cdot \mathrm{h}^{-1}$ when the PVA/xylan weight ratio was $2: 1\left(\mathrm{P} 2 \mathrm{X1}\right.$ ), and the highest value was $0.054 \mathrm{~g} \cdot \mu \mathrm{m} \cdot \mathrm{cm}^{-2} \cdot \mathrm{h}^{-1}$ at the PVA/xylan weight ratio of $1: 2$ (P1X2). These results could be attributed to the changes in the functionality properties of the blending films prepared in the different PVA/xylan weight ratio. Clearly, BTCA had a remarkable influence on WVP. When the BTCA content was 5\%, WVP of PVA/xylan blending films had the lowest values for $0.015 \mathrm{~g} \cdot \mu \mathrm{m} \cdot \mathrm{cm}^{-2} \cdot \mathrm{h}^{-1}$ at the PVA/xylan weight ratio of $3: 1$. This meant that WVP decreased with addition of a little amount of BTCA. This result might be attributed to the water-resistant nature of BTCA. Nevertheless, the WVP increase was directly proportional to the BTCA content in the range of $10 \%-25 \%$ and then incremented sharply up to the highest values of $0.0274 \mathrm{~g} \cdot \mu \mathrm{m} \cdot \mathrm{cm}^{-2} \cdot \mathrm{h}^{-1}$ at the content of $30 \%$ BTCA. The

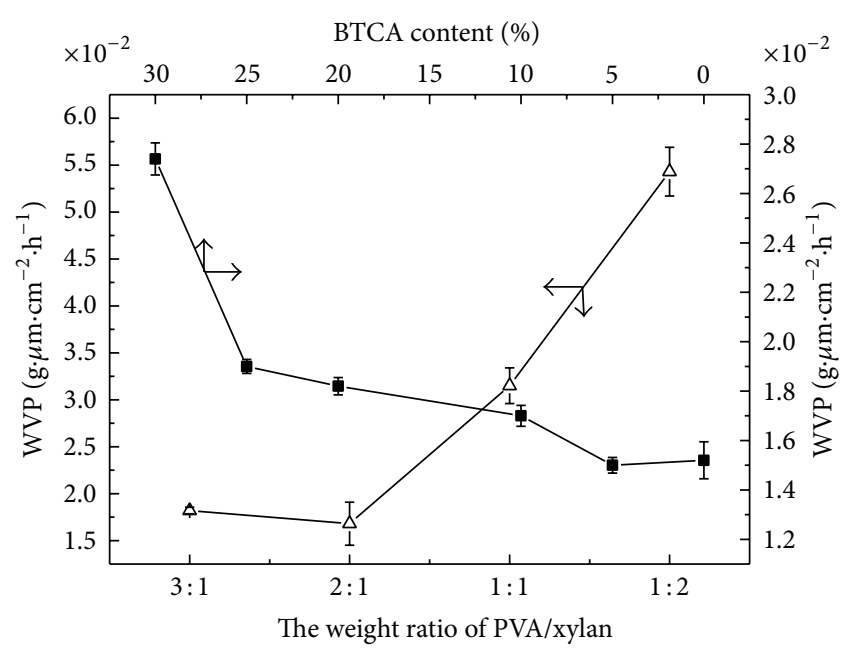

FIGURE 3: The effect of the weight ratio of PVA/xylan and the BTCA content on WVP.

TABLE 2: The WA, $S$, and WC of all the PVA/xylan blending films.

\begin{tabular}{lccc}
\hline Samples & WA & $S(\%)$ & WC $(\%)$ \\
\hline P3X1 & 0.45 & 19.16 & 8.53 \\
P2X1 & 0.62 & 22.35 & 10.53 \\
P1X1 & 0.84 & 26.38 & 11.65 \\
P1X2 & 0.93 & 30.15 & 12.31 \\
PX0 & 1.30 & 37.29 & 7.44 \\
PX5 & 1.18 & 26.74 & 11.62 \\
PX10 & 1.07 & 26.76 & 16.59 \\
PX25 & 0.34 & 25.51 & 9.12 \\
PX30 & 0.31 & 31.22 & 9.27 \\
\hline
\end{tabular}

higher plasticizer content resulted in more permeable films [27]. Mikkonen et al. [12] gained the same conclusions. This may be due to the formation of intermolecular interactions of BTCA and polyhydroxylic compounds with the increase of BTCA content.

As reported in the literature, García et al. [28] speculated many factors such as polymeric chain mobility and specific interaction between the functional groups of the polymers had effects on WVP. The decrease of WVP may be due to the formation of new interchain interaction. Ghasemlou et al. [29] decreased WVP and improved barrier properties of starch films up to $50 \%$ relative to the control sample by adding Zataria multiflora Boiss (ZEO) or Mentha pulegium (MEO). They thought that the hydrogen and covalent interactions between the starch network and these polyphenolic compounds might limit the availability of hydrogen groups to form hydrophilic bonds with water.

3.3. The WA, $S$, and WC Determination. The WA, $S$, and WC of all the PVA/xylan blending films are shown in Table 2. These data were used to further analyze barrier properties of blending films according to the experimental design previously presented (Table 1). When the BTCA content 
maintained $20 \%$, the WA increased gradually with the weight ratio of PVA and xylan from $3: 1$ to $1: 2$. Comparatively, the highest WA value reached to 1.30 for the blending films in the PVA/xylan weight ratio of $3: 1$ without the addition of BTCA. When the weight ratio of PVA and xylan was $3: 1$, WA values were decreased along with BTCA amount increasing from $5 \%$ to $30 \%$. So the addition of BTCA could efficiently reduce WA of blending films. It was due to increasing moisture barrier properties with addition of BTCA. The solubility of blending films is a crucial influencing factor for potential application such as food packaging. In most cases, water solubility should be lower in order to improve the water resistance and integrity of films, especially for packaging foods [30]. As we can see from Table 2, water solubility increased steadily from $19.16 \%$ (P3X1) to $30.15 \%$ (P1X2) with 20\% BTCA. The increase of the solubility can be explained by the increase of content of hydroxyl groups with the increase of xylan in the blending films. The weight ratio of PVA and xylan was chosen for $3: 1$ which was superior to others as the solubility decreased up to $19.16 \%$, lower than results of $20 \%$ solubility studied by Alves et al. [18]. Then $S$ decreased with increasing BTCA content from $0 \%$ to $25 \%$. However, when BTCA content reached $30 \%$, the water solubility had a sudden increase which might be due to increment of hydrophilicity of blending films with high concentration of BTCA. Additionally, water content increased gradually with increasing weight ratio of PVA and xylan. For plasticized films, the addition of $20 \%$ BTCA to blending films (P3X1) was able to obtain relatively lower water content for $8.53 \%$. This is interpreted as a result of interactions between BTCA and the PVA/xylan composite film matrix, which reduces in the film's affinity for water and limits the accessibility of water molecules in the films [31]. With a further increase of BTCA concentration, the film matrix exposed more hydroxyl groups and increased water uptake. Unplasticized samples had lower water uptake (7.44\%).

3.4. Thermogravimetric Analysis (TGA). Thermogravimetric analysis is a standard technique to determine the composition or thermal stability of materials. The results of TGA and DTG curves of PVA/xylan blending films with different BTCA contents and PVA/xylan weight ratios are illustrated in Figure 4. The pure PVA/xylan films (PX0) exhibited apparently two-step degradation. With the degradation of PVA and xylan chains, the first step region occurred at around $250-330^{\circ} \mathrm{C}$, and then carbonization of polymer matrix at the high temperature around $390-470^{\circ} \mathrm{C}$ formed the second degradation region. In contrast, the BTCA-plasticized films (P3X1, P1X1, and PX10) had three-step degradation process without obvious demarcation line. Four degradations peaks for PX0, P3X1, PX10, and P1X1 were observed at 316, 441, 361 , and $433^{\circ} \mathrm{C}$, respectively. The sudden drop in weight with increasing temperature occurred between 250 and $400^{\circ} \mathrm{C}$. The decrease of hydrogen bonds may lead to the decreasing thermal stability of films, because the rupture of hydrogen bonds requires more energy during thermal degradation [6]. As expected, the total weight loss of the plasticized PVA/xylan films was lower than that of pure composite film (PX0).

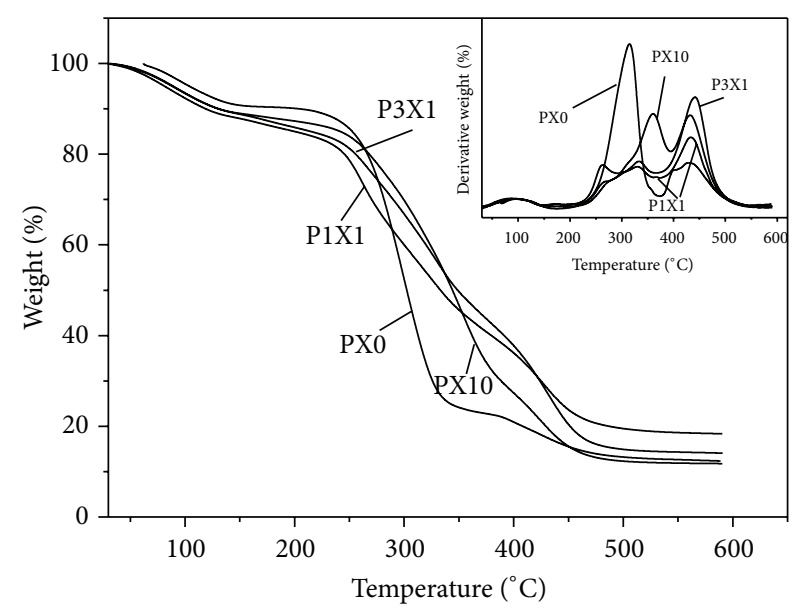

FIGURE 4: TGA and DTGA curves of PVA/xylan blending films.

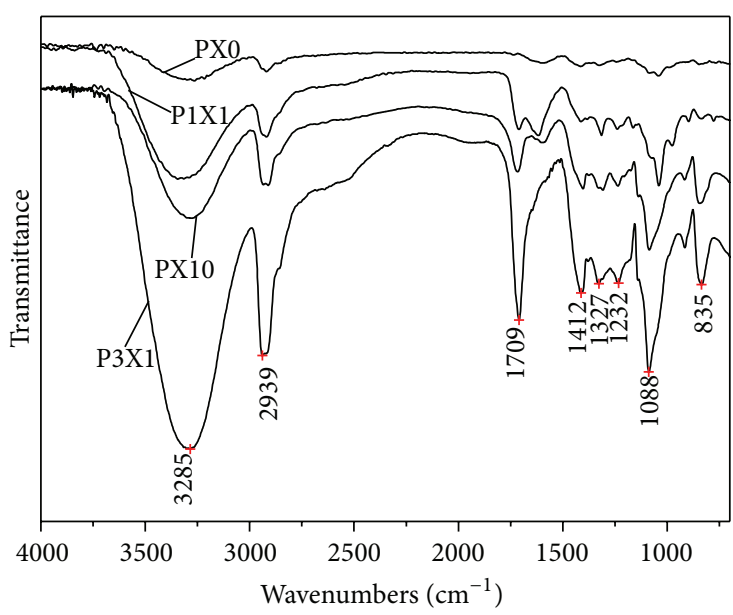

FIGURE 5: FTIR spectra of PVA/xylan blending films.

This might be due to the incorporation of the BTCA which stabilized the composites at high temperature.

3.5. FTIR Spectroscopy. FTIR spectroscopy was used to examine the interactions between PVA/xylan and BTCA. The broad band at $3285 \mathrm{~cm}^{-1}$ originates from the stretching of $-\mathrm{OH}$ groups; this peak was larger than the film PX0 due to overlapping stretching of hydrogen bonds, $\mathrm{O}-\mathrm{H}$ stretching of the composite films [32], which shifts $22 \mathrm{~cm}^{-1}$ compared with that of unplasticized PVA/xylan $\left(3263 \mathrm{~cm}^{-1}\right)$. It indicated that BTCA interacted with PVA/xylan films matrix, and the hydroxyl groups between PVA and xylan were partially destructed [33]. And the peak at $2939 \mathrm{~cm}^{-1}$ is assigned to $\mathrm{CH}$ - stretching. A sharp brand at $1709 \mathrm{~cm}^{-1}$ is the $\mathrm{C}=\mathrm{O}$ bond and suggested the presence of a carbonyl group mainly from BTCA in the film matrix and the $\mathrm{C}=\mathrm{O}$ bond stretching was stronger with the increase of BTCA which had similar trend with $-\mathrm{OH}$ stretching. The absorption bands at $1412 \mathrm{~cm}^{-1}$ in $\mathrm{P} 3 \mathrm{X} 1$ can be attributed to $\mathrm{O}-\mathrm{H}, \mathrm{C}-\mathrm{H}$ bending from PVA structure and the absorption bands at $1327 \mathrm{~cm}^{-1}, 1232 \mathrm{~cm}^{-1}$ 


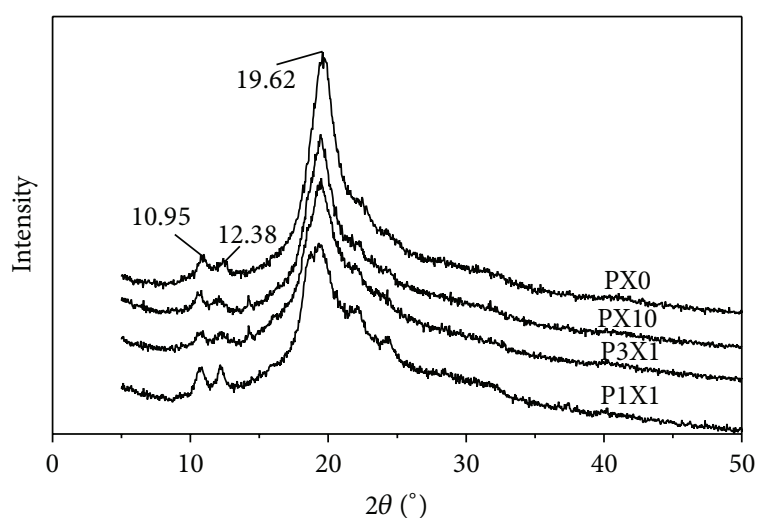

Figure 6: X-ray diffraction of PVA/xylan blending films.

to $\delta(\mathrm{OH})$ with $\mathrm{C}-\mathrm{H}$ wagging, respectively. The brands from 835 to $1088 \mathrm{~cm}^{-1}$ corresponded to the $\mathrm{C}-\mathrm{O}$ bond stretching.

3.6. X-Ray Diffraction. X-ray diffractograms of PVA/xylan blending films are shown in Figure 6. As observed, three main diffraction peaks $\left(2 \theta\right.$ about $10.95^{\circ}, 12.38^{\circ}$, and $\left.19.62^{\circ}\right)$ were seen in the X-ray diffraction pattern. The unplasticized films had the highest intensity values corresponding to different regions. When the BTCA content increased from $10 \%$ to $20 \%$, the intensity of crystalline peaks decreased a little and the diffraction patterns were quite similar and showed no significant differences with the presence of plasticizer (BTCA). However, the peak became wider and amorphous when the weight ratio of PVA and xylan reduced to $1: 1$. Furthermore, under this condition, two new peaks appeared at $2 \theta$ between $20^{\circ}$ and $25^{\circ}$. These changes demonstrated an interaction between the components. The interaction restricted the mobility of the polymer chains and may lead to a considerable slowing of the recrystallization of the matrix during the drying process. This changes the crystalline structure of the composite films. Crystallinity analysis indicated that the xylan in the samples was semicrystalline [34]. Peng et al. [35] also found that the xylan films were semicrystalline.

3.7. Film Deterioration in Soil. The current study focused on increasing biocompatibility and degradability of blending films by adding biodegradable biopolymers. All the films had the same size and shape to prevent the external effects on biodegradation of films [36]. The dependence of degradation of PVA/xylan blending films with different BTCA contents as a function of time is presented in Figure 7. The test was carried out for 30 days of burial in soil under prevailing environmental conditions. For unplasticized films (PX0), the biggest characteristic of their decomposition rate was faster than plasticized films (PX10, PX30) in a beginning dozen days and then the weight loss gradually fell behind those that were plasticized by BTCA. After deterioration in soil about 12 days, blending films with 10\% BTCA had higher weight loss ratio and showed a clear advantage. When the films were deteriorated for half a month, weight loss of PX10 reached 31.7\%, while PX0 and PX30 were 28.5\%, 26.6\%,

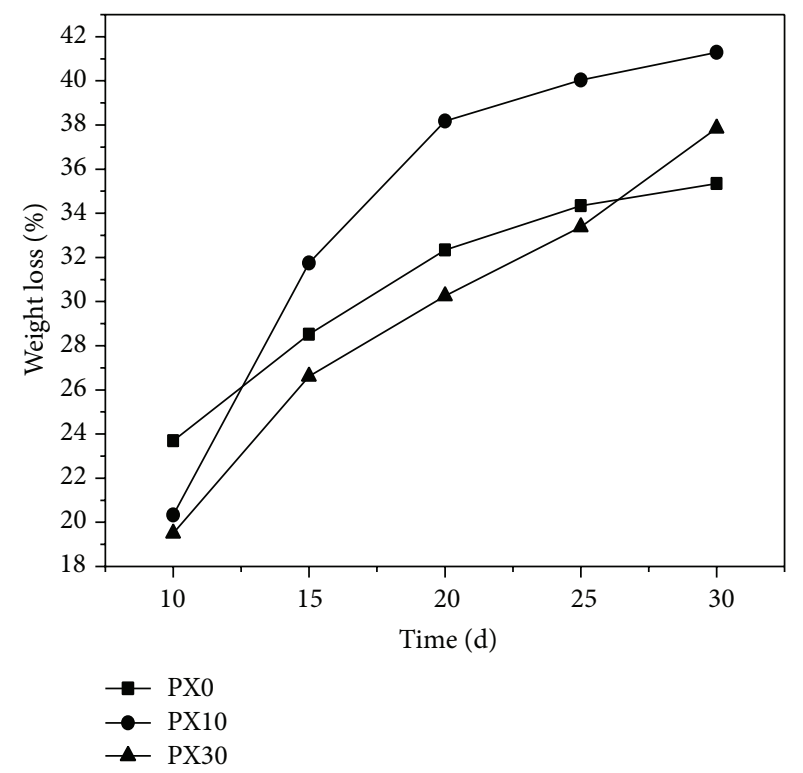

Figure 7: The effect of the different BTCA contents on the degradation of PVA/xylan blending films.

respectively. The weight loss of the PX30 films increased by $13.4 \%$ during the last 5 days in a month and rapidly surpassed the PX0 films. Eventually, the PX10 films were biodegraded by $41.29 \%$ within 30 days and the degree of disintegration was considerable. Chai et al. [16] investigated effects of the content of modified starch on the biodegradation of the PVA/modified starch blending films and the optimal degree of degradation was obviously below $40 \%$ within 30 days. The films with $30 \%$ BTCA also achieved $37.8 \%$, while the weight loss of untreated films (PX0) was 35.3\%, inferior to the plasticized films. Therefore, the addition of BTCA had a positive influence on degradation of PVA/xylan blending films. Imam et al. [37] explained that the addition of a plasticizer in formulations lowered the overall extent of degradation in composites. Also, biopolymers themselves like xylan have stronger biocompatibility and stimulate PVA degradation in soil.

\section{Conclusions}

PVA/xylan blending films by casting method were successfully synthesized by using BTCA as a new plasticizer. It was found that TS and EAB values reduced with the weight ratio of PVA/xylan from $3: 1$ to $1: 2$. Additionally, under the condition of optimal weight ratio of PVA/xylan for $3: 1$, both TS and EAB increased firstly and then decreased along with the increasing BTCA content. WVP was decreased first and then increased sharply with the decreasing weight ratio of PVA/xylan. The effect of BTCA content on WVP had similar tendency. The WA values were decreased along with increasing BTCA amount due to moisture barrier properties with addition of BTCA. The thermal stability of the plasticized blending films had improved. FTIR and XRD indicated the presence of interactions and structural changes between 
BTCA and PVA/xylan matrix. During deterioration in soil for 30 days, PVA/xylan blending films achieved $41.3 \%$ weight loss. In summary, the PVA/xylan blending films performed desired mechanical properties and excellent biocompatibility and biodegradation, which indicate that PVA/xylan blending films have promising applications for food packaging and agricultural mulch owing to their sustainable and ecofriendly features.

\section{Conflict of Interests}

The authors declare that there is no conflict of interests regarding the publication of this paper.

\section{Acknowledgments}

This work was supported by Grants from National Natural Science Foundation of China (no. 31070530), the Author of National Excellent Doctoral Dissertation of China (201169), Guangdong Natural Science Funds for Distinguished Young Scholar (S20120011250), Science and Technology Project of Guangdong Province (2011B050400015), and the Fundamental Research Funds for the Central Universities (2014ZG0003), SCUT (South China University of Technology).

\section{References}

[1] M. Jawaid and H. P. S. Abdul Khalil, "Cellulosic/synthetic fibre reinforced polymer hybrid composites: a review," Carbohydrate Polymers, vol. 86, no. 1, pp. 1-18, 2011.

[2] Y. X. Xu, K. M. Kim, M. A. Hanna, and D. Nag, "Chitosan-starch composite film: preparation and characterization," Industrial Crops and Products, vol. 21, no. 2, pp. 185-192, 2005.

[3] Z. Merali, J. D. Ho, S. R. A. Collins et al., "Characterization of cell wall components of wheat straw following hydrothermal pretreatment and fractionation," Bioresource Technology, vol. 131, pp. 226-234, 2013.

[4] N. M. L. Hansen and D. Plackett, "Sustainable films and coatings from hemicelluloses: a review," Biomacromolecules, vol. 9, no. 6, pp. 1493-1505, 2008.

[5] K. S. Mikkonen and M. Tenkanen, "Sustainable food-packaging materials based on future biorefinery products: xylan and mannans," Trends in Food Science \& Technology, vol. 28, no. 2, pp. 90-102, 2012.

[6] L. X. Zhong, X. W. Peng, D. Yang, X. F. Cao, and R. C. Sun, "Long-chain anhydride modification: a new strategy for preparing xylan films," Journal of Agricultural and Food Chemistry, vol. 61, no. 3, pp. 655-661, 2013.

[7] Z. A. N. Hannai, J. McNamara, Y. H. Roos, and J. P. Kerry, "Effect of plasticizer content on the functional properties of extruded gelatin-based composite films," Food Hydrocolloids, vol. 31, no. 2, pp. 2642-2669, 2013.

[8] N. Cao, X. Yang, and Y. Fu, "Effects of various plasticizers on mechanical and water vapor barrier properties of gelatin films," Food Hydrocolloids, vol. 23, no. 3, pp. 729-735, 2009.

[9] J. Piermaria, A. Bosch, A. Pinotti, O. Yantorno, M. A. Garcia, and A. G. Abraham, "Kefiran films plasticized with sugars and polyols: water vapor barrier and mechanical properties in relation to their microstructure analyzed by ATR/FT-IR spectroscopy," Food Hydrocolloids, vol. 25, no. 5, pp. 1261-1269, 2011.

[10] M. J. Fabra, P. Talens, R. Gavara, and A. Chiralt, "Barrier properties of sodium caseinate films as affected by lipid composition and moisture content," Journal of Food Engineering, vol. 109, no. 3, pp. 372-379, 2012.

[11] M. S. Peresin, K. Kammiovirta, H. Setala, and T. Temmelin, "Structural features and water interactions of etherified xylan thin films," Journal of Polymers and the Environment, vol. 20, no. 4, pp. 895-904, 2012.

[12] K. S. Mikkonen, S. Heikkinen, A. Soovre et al., "Films from oat spelt arabinoxylan plasticized with glycerol and sorbitol," Journal of Applied Polymer Science, vol. 114, no. 1, pp. 457-466, 2009.

[13] E. Chiellini, P. Cinelli, E. G. Fernandes, E.-R. S. Kenawy, and A. Lazzeri, "Gelatin-based blends and composites. Morphological and thermal mechanical characterization," Biomacromolecules, vol. 2, no. 3, pp. 806-811, 2001.

[14] C.-C. Yang, "Synthesis and characterization of the cross-linked $\mathrm{PVA} / \mathrm{TiO}_{2}$ composite polymer membrane for alkaline DMFC," Journal of Membrane Science, vol. 288, no. 1-2, pp. 51-60, 2007.

[15] P.-Y. Zhuang, Y.-L. Li, L. Fan, J. Lin, and Q.-L. Hu, "Modification of chitosan membrane with poly(vinyl alcohol) and biocompatibility evaluation," International Journal of Biological Macromolecules, vol. 50, no. 3, pp. 658-663, 2012.

[16] W.-L. Chai, J.-D. Chow, and C.-C. Chen, "Effects of modified starch and different molecular weight polyvinyl alcohols on biodegradable characteristics of polyvinyl alcohol/starch blends," Journal of Polymers \& the Environment, vol. 20, no. 2, pp. 550-564, 2012.

[17] S.-D. Yoon, M.-H. Park, and H.-S. Byun, "Mechanical and water barrier properties of starch/PVA composite films by adding nano-sized poly(methyl methacrylate-co-acrylamide) particles," Carbohydrate Polymers, vol. 87, no. 1, pp. 676-686, 2012.

[18] P. M. A. Alves, R. A. Carvalho, I. C. F. Moraes, C. G. Luciano, A. M. Q. B. Bittante, and P. J. A. Sobral, "Development of films based on blends of gelatin and poly(vinyl alcohol) cross linked with glutaraldehyde," Food Hydrocolloids, vol. 25, no. 7, pp. 17511757, 2011.

[19] M. Montazer, P. Golshani, and M. B. Moghadam, "Hydrophobic, cross-linked and photoactive cotton fabric using nano $\mathrm{TiO}_{2}$ and BTCA-statistically optimized by RSM," Indian Journal of Fibre \& Textile Research, vol. 38, pp. 35-43, 2013.

[20] J.Zoldners and T. Kiseleva, "Modification of Hemicellulose with polycarboxylic acids," Holzforschung, vol. 67, no. 5, pp. 567-571, 2013.

[21] W. Huang, Y. Song, Y. Xing, and J. Dai, "Durable hydrophobic cellulose fabric prepared with polycarboxylic acid catalyzed silica sol," Industrial and Engineering Chemistry Research, vol. 49, no. 19, pp. 9135-9142, 2010.

[22] B. S. Kayserilioğlu, W. M. Stevels, W. J. Mulder, and N. Akkas, "Mechanical and biochemical characterization of wheat gluten films as a function of $\mathrm{pH}$ and co-solvent," Starch/Starke, vol. 53, no. 8, pp. 381-386, 2001.

[23] A. Ebringerová and T. Heinze, "Xylan and xylan derivativesbiopolymers with valuable properties," Macromolecular Rapid Communications, vol. 21, no. 9, pp. 542-556, 2000.

[24] M. Mohsin, A. Hossin, and Y. Haik, "Thermomechanical properties of poly(vinyl alcohol) plasticized with varying ratios of sorbitol," Materials Science and Engineering A, vol. 528, no. 3, pp. 925-930, 2011. 
[25] F. E. F. Silva, M. C. B. Di-Medeiros, K. A. Batista, and K. F. Fernandes, "PVA/polysaccharides blended films: mechanical properties," Journal of Materials, vol. 2013, Article ID 413578, 6 pages, 2013.

[26] S.-D. Yoon, S.-H. Chough, and H.-R. Park, "Effects of additives with different functional groups on the physical properties of starch/PVA blend film," Journal of Applied Polymer Science, vol. 100, no. 5, pp. 3733-3740, 2006.

[27] I. S. Arvanitoyannis, A. Nakayama, and S.-I. Aiba, "Chitosan and gelatin based edible films: state diagrams, mechanical and permeation properties," Carbohydrate Polymers, vol. 37, no. 4, pp. 371-382, 1998.

[28] M. A. García, M. N. Martino, and N. E. Zaritzky, "Lipid addition to improve barrier properties of edible starch-based films and coatings," Journal of Food Science, vol. 65, no. 6, pp. 941-944, 2000.

[29] M. Ghasemlou, N. Aliheidari, R. Fahmid et al., "Physical, mechanical and barrier properties of corn starch films incorporated with plant essential oils," Carbohydrate Polymers, vol. 98, no. 1, pp. 1117-1126, 2013.

[30] S. F. Hosseini, M. Rezaei, M. Zandi, and F. F. Ghavi, "Preparation and functional properties of fish gelatin-chitosan blend edible films," Food Chemistry, vol. 136, no. 3-4, pp. 1490-1495, 2013.

[31] L. Higueras, G. Lopez-Carballo, J. P. Cerisuelo, R. Gavara, and P. Hernandez-Munoz, "Preparation and characterization of chitosan/HP- $\beta$-cyclodextrins composites with high sorption capacity for carvacrol," Carbohydrate Polymers, vol. 97, no. 2, pp. 262-268, 2013.

[32] K. S. V. K. Rao, B. Vijaya Kumar Naidu, M. C. S. Subha, M. Sairam, and T. M. Aminabhavi, "Novel chitosan-based pHsensitive interpenetrating network microgels for the controlled release of cefadroxil," Carbohydrate Polymers, vol. 66, no. 3, pp. 333-344, 2006.

[33] D. Huang, B. Mu, and A. Wang, "Preparation and properties of chitosan/poly (vinyl alcohol) nanocomposite films reinforced with rod-like sepiolite," Materials Letters, vol. 86, pp. 69-72, 2012.

[34] S. L. Heikkinen, K. S. Mikkonen, K. Pirkkalainen, R. Serimaa, C. Joly, and M. Tenkanen, "Specific enzymatic tailoring of wheat arabinoxylan reveals the role of substitution on xylan film properties," Carbohydrate Polymers, vol. 92, no. 1, pp. 733-740, 2013.

[35] X.-W. Peng, J.-L. Ren, L.-X. Zhong, and R.-C. Sun, "Nanocomposite films based on xylan-rich hemicelluloses and cellulose nanofibers with enhanced mechanical properties," Biomacromolecules, vol. 12, no. 9, pp. 3321-3329, 2011.

[36] H.-S. Yang, J.-S. Yoon, and M.-N. Kim, "Dependence of biodegradability of plastics in compost on the shape of specimens," Polymer Degradation and Stability, vol. 87, no. 1, pp. 131135, 2005.

[37] S. H. Imam, P. Cinelli, S. H. Gordon, and E. Chiellini, "Characterization of biodegradable composite films prepared from blends of poly(vinyl alcohol), cornstarch, and lignocellulosic fiber," Journal of Polymers and the Environment, vol. 13, no. 1, pp. 47-55, 2005. 

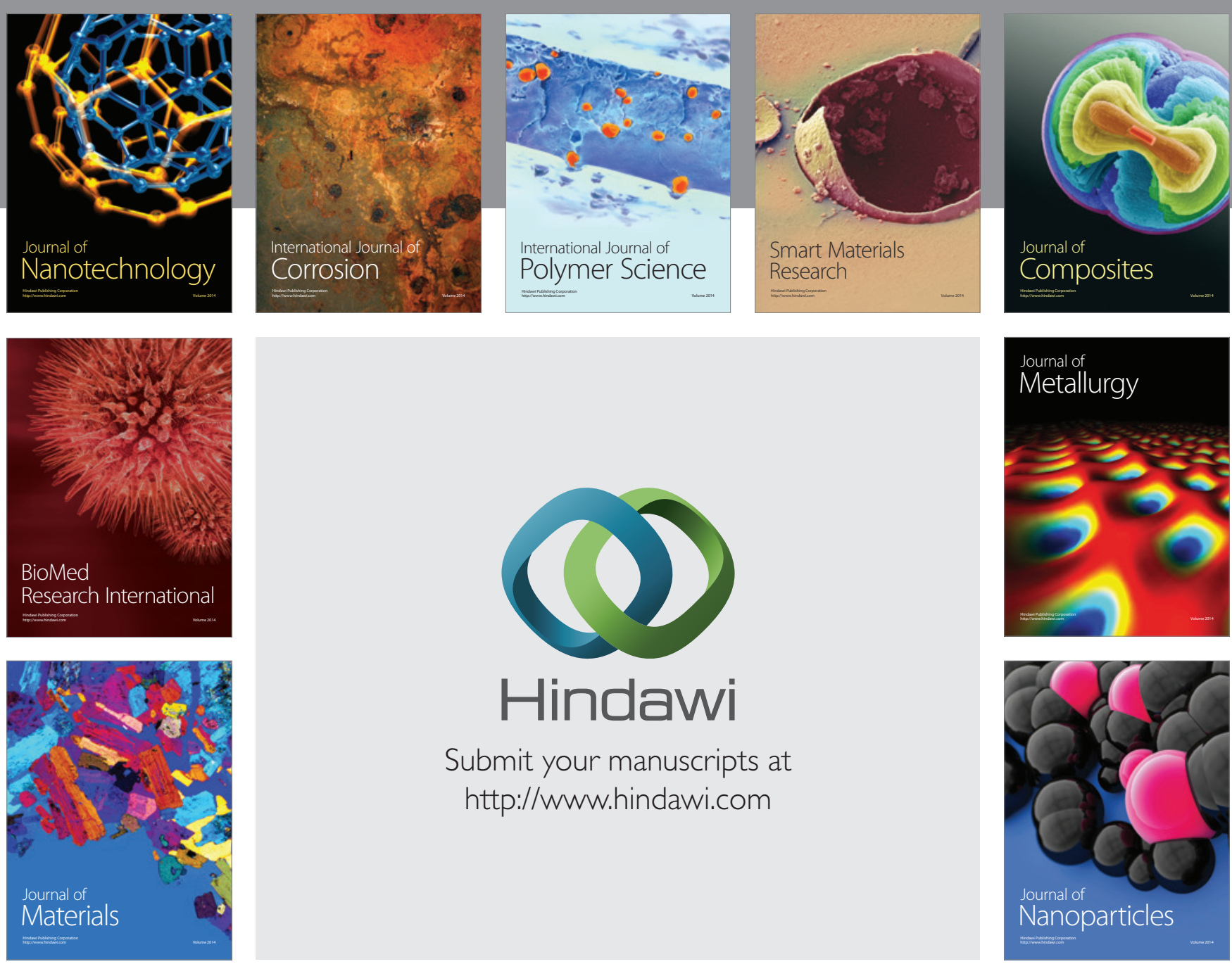

Submit your manuscripts at http://www.hindawi.com
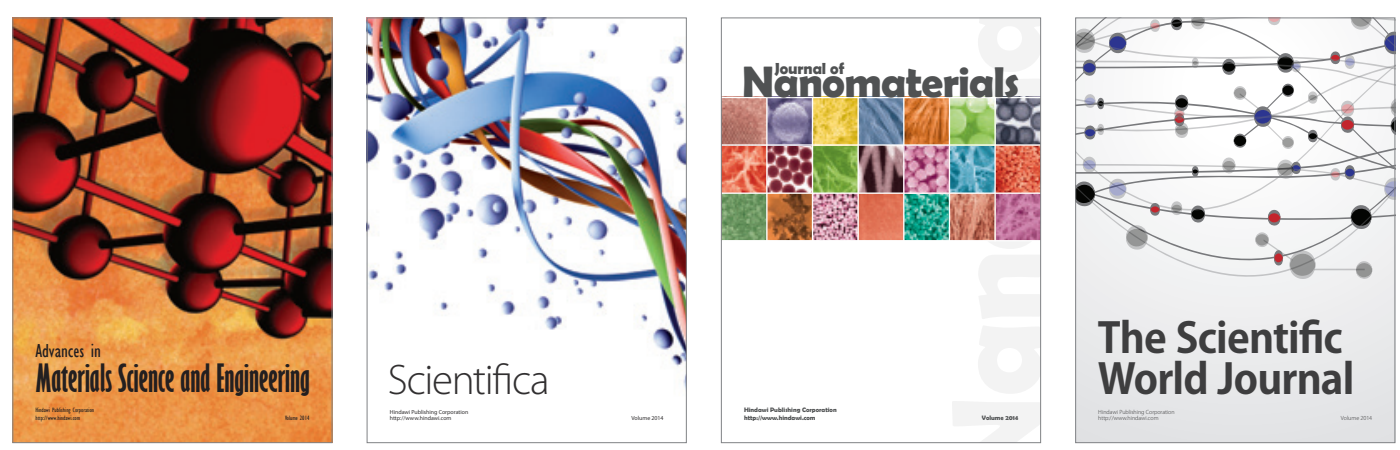

\section{The Scientific World Journal}
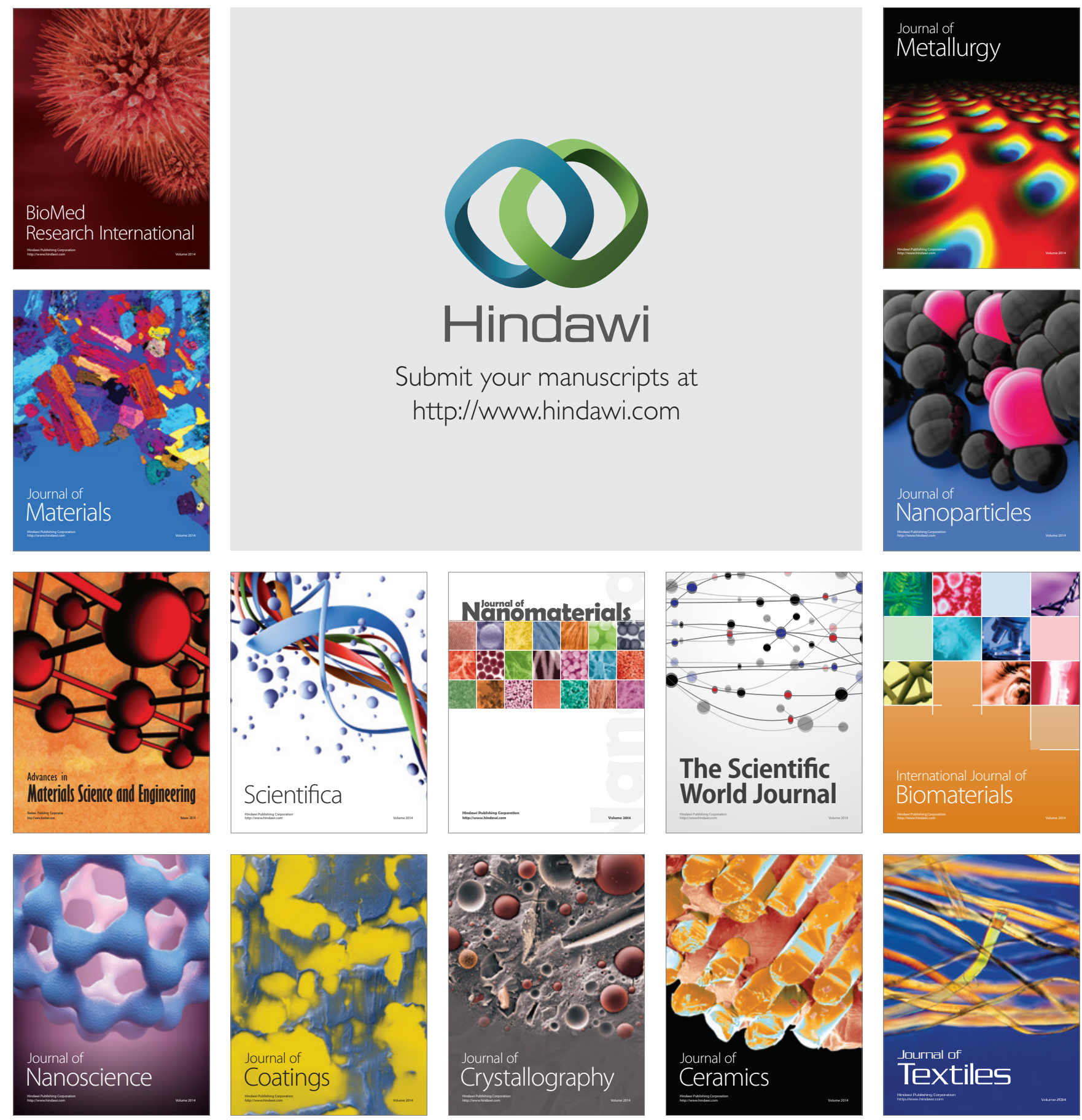